\title{
The Study of Sound Scattering Structures for the Purposes of Room Acoustic Enhancement
}

\author{
T. Kamisiński, J. Rubacha And A. PilCh \\ Department of Mechanics and Vibroacoustics, University of Science and Technology - AGH \\ al. Mickiewicza 30, 30-095 Kraków, Poland
}

\begin{abstract}
Acoustic structures are currently classified mainly in terms of their acoustic absorption and insulation properties. Knowing the sound scattering parameter can significantly improve the useful value of materials and identify their best applications. Currently no studies are performed in Poland on the sound scattering coefficients of materials. This is due to a complex measurement procedure and a lack of legal requirements. The authors have attempted to make such measurements on the basis of the standard ISO 17497-1:2004: Acoustics Sound-scattering properties of surfaces - Part 1: Measurement of the random-incidence scattering coefficient in a reverberation room. Measurement and calculation methods are presented, and problems encountered during this study have been described. This issue is of particular importance, especially in the acoustic design of interiors.
\end{abstract}

PACS numbers: 43.20.Fn, 43.55.Br, 43.58.+z

\section{Introduction}

In recent years, increased interest in the study and designing of sound scattering systems has been noted. This issue is particularly important in the acoustic modelling of interiors. Knowing the scattering parameter allows for the proper use of materials for correcting acoustic deficiencies in interiors and in shaping the acoustic fields. Up to now, such studies have not been conducted in Poland, due to a complex measurement procedure and a lack of legal requirements. Acoustic systems have been mainly classified according to their sound absorption and insulation properties.

So far, the ISO 17497-1:2004 [1] standard has been released, which defines the scattering coefficient $s$ and the method of its determination in the reverberation chamber. The coefficient defines in a quantitative manner the scattering properties of the surface. In qualitative terms, the surface is defined by the sound diffusion coefficient $d$, which, along with the method of its determination, is defined by the draft ISO 17497-2 standard. The diffusion coefficient $d_{\theta}$ provides information on how uniformly the reflected energy is distributed for a given angle of incidence. This paper describes the scattering coefficient $s$, the methods of determining it in the reverberation chamber and the measurement results obtained for a prototype scattering structure.

\section{Determination of the scattering coefficient}

The energy of the incident acoustic wave is distributed in three ways: one part is converted into heat inside the material it falls upon, and the amount of this transformed energy defines the sound absorption properties of the material. Another part of the energy passes through the material, which depends on its sound insulation properties, whereas the remaining part of energy is reflected from the surface and is returned to the surroundings. In the simplest case, it is a specular reflection, where Snell's law is obeyed, which means that the angle of reflection is equal to the angle of incidence and takes place in the same plane. The energy reflected at angles other than the angle of incidence is called scattered energy. The amount of this energy is defined by the scattering coefficient, which is defined by the formula

$$
s=1-\frac{E_{\mathrm{spec}}}{E_{\mathrm{tot}}},
$$

where: $s$ is the scattering coefficient, $E_{\text {spec }}$ is the energy of specularly reflected sound, $E_{\mathrm{tot}}$ is the total reflected energy.

The scattering coefficient $s$ characterises the scattering properties of a given structure in a quantitative manner. It can assume values from 0 to 1 , where 0 represents a surface producing a fully specular reflection, whereas 1 corresponds to a fully scattering surface [2].

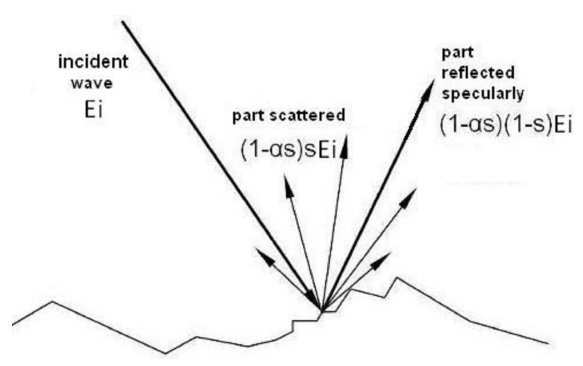

Fig. 1. Graphical representation of the sound scattering coefficient $s$ (after Cox [3]). 
The idea of the measurement of the scattering coefficient $s$ consists in determining the amount of energy reflected specularly from a given surface. Based in Fig. 1, the part of energy reflected specularly can be determined from the formula:

$$
E_{\text {spec }}=\left(\left(1-\alpha_{s}\right)(1-s)\right) E_{i} \equiv\left(1-\alpha_{\text {spec }}\right) E_{i},
$$

where: $E_{i}$ is the incident wave energy; $E_{\mathrm{spec}}$ is the part of energy reflected specularly; $s$ is the scattering coefficient; $\alpha_{s}$ is the sample's absorption coefficient and $\alpha_{\text {spec }}$ is the specular absorption coefficient.

The amount of energy absorbed at specular reflection is characterized by the specular absorption coefficient $\alpha_{\text {spec }}$. This coefficient accounts for the amount of energy absorbed at specularly reflected sound, which results from the sound absorption properties of the material, and sound reflected at directions other than specular. If this coefficient and the reverberation absorption coefficient $\alpha_{s}$ are known, one can determine the scattering coefficient $s$ from the equation:

$$
s=1-\frac{1-\alpha_{\text {spec }}}{1-\alpha_{s}}=\frac{\alpha_{\text {spec }}-\alpha_{s}}{1-\alpha_{s}} .
$$

Sound absorption coefficients are determined from the formulae given below:

- the sample's reverberation absorption coefficient

$$
\alpha_{s}=55.3 \frac{V}{S}\left(\frac{1}{c_{2} T_{2}}-\frac{1}{c_{1} T_{1}}\right)-4 \frac{V}{S}\left(m_{2}-m_{1}\right),
$$

where: $V$ is the volume of the reverberation chamber $\left(\mathrm{m}^{3}\right) ; S$ is the surface area of the studied sample $\left(\mathrm{m}^{2}\right)$; $T_{1}$ is the reverberation time for the stationary base plate of the turntable; $T_{2}$ is the reverberation time of the sample positioned on the turntable; $c_{1}$ is the speed of sound during the measurement of $T_{1} ; c_{2}$ is the speed of sound during the measurement of $T_{2} ; m_{1}, m_{2}$ are the energy attenuation coefficients of air $(1 / \mathrm{m})$ during the measurement of $T_{1}$ and $T_{2}$ (ISO 9613-1).

- sample's specular absorption coefficient $\alpha_{\mathrm{spec}}$

$$
\alpha_{\text {spec }}=55.3 \frac{V}{S}\left(\frac{1}{c_{4} T_{4}}-\frac{1}{c_{3} T_{3}}\right)-4 \frac{V}{S}\left(m_{4}-m_{3}\right),
$$

where: $T_{3}$ is the reverberation time for the turntable's rotating base plate; $T_{4}$ is the reverberation time for the sample rotating on the turntable; $c_{3}$ is the speed of sound during the measurements of $T_{3} ; c_{4}$ is the speed of sound during the measurements of $T_{4} ; m_{3}, m_{4}$ are the energy attenuation coefficient of air $(1 / \mathrm{m})$ during the measurement of $T_{1}$, and $T_{2}, T_{3}, T_{4}$, (ISO 9613-1).

\section{Acoustic measurements}

The measurement of the scattering coefficient $s$, is performed in the reverberation chamber. The measurement system consists of a turntable on which the sample studied is mounted, a measurement microphone, an omnidirectional sound source, a measurement signal generator, and a signal analyser (Fig. 2). During the measurement impulse responses are determined for four measurement series: for a rotating and stationary turntable with and without the sample. Next, by integrating the impulse responses, four reverberation times are obtained which are used to determine the reverberation absorption coefficient $\alpha_{s}$ and the specular absorption coefficient $\alpha_{\text {spec }}$.

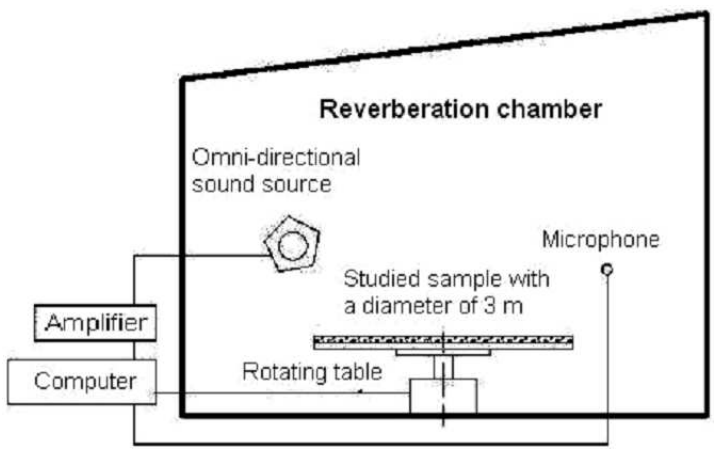

Fig. 2. Block diagram of the measurement system for sound scattering coefficient, $s$.

The measurement of the scattering coefficient $s$ was performed on a sample based on the $N=7$ quadratic residue sequence. Elements of the sequence $s_{n}$ were found from the formula

$$
s_{n}=n^{2} \bmod N \text {, }
$$

where: $n$ are the successive non-negative integers $(0,1$, $\ldots, n-1) ; N$ is the number of wells per period.

The depth of the wells $d_{n}$ of the system studied is

$$
d_{n}=\frac{s_{n} \lambda_{0}}{2 N}
$$

where: $\lambda_{0}$ is the wavelength at the design frequency $f_{0}$.

The design frequency $f_{0}$ is taken as a lower limit for the diffuser system. The upper limit depends on the well width, which is found from the formula:

$$
w=\frac{\lambda_{\min }}{2},
$$

where: $\lambda_{\min }$ is the wavelength at the upper frequency limit.

Figure 3 presents impulse responses for three various positions of the sample shown in Figs. 4 and 5 for the same positions of the sound source and the microphone. It can be seen that in the initial time interval the responses are the same, which corresponds to the sound directly reaching the microphone and the wave specularly reflected from the sample and the chamber walls (Fig. 3a). Later, the responses begin to differ, which indicates the presence of scattered reflections, that is those from directions other than specular. Figure $3 \mathrm{~b}$ show the responses for later time intervals, where there is no correlation among the signals. The response averaged over the complete rotation of the sample has a reduced section with scattered reflections. Based on the reverberation times found from impulse responses averaged over the sample rotation, the coefficient of specular absorption $\alpha_{\text {spec }}$ was calculated.

A computer with an external sound card was used in the measurements, which allowed measurement signals to be generated and recorded. The transmission line con- 


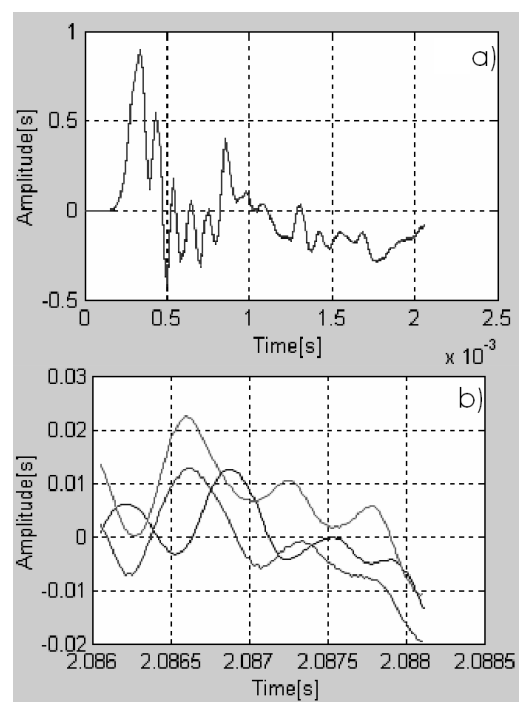

Fig. 3. Impulse responses for three positions of the sample.

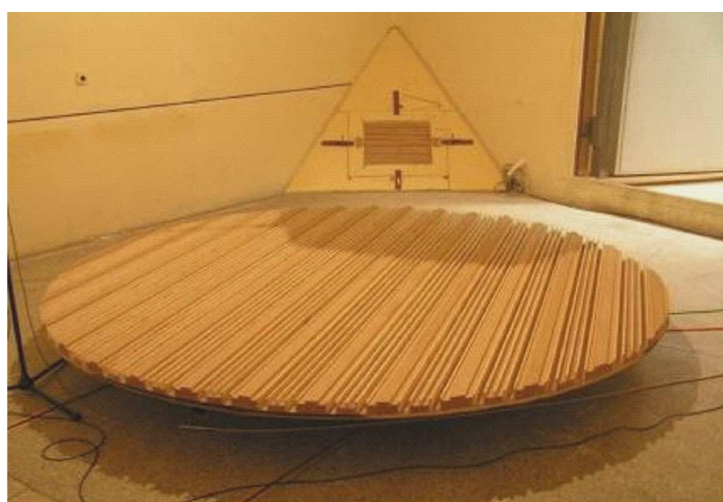

Fig. 4. A sample in the measurement system in a reverberation chamber.

sisted of a power amplifier feeding the omnidirectional sound source. The receiver line consisted of two measurement microphones and the preamplifiers. A special software, called Dirac [4], was used to record the impulse responses and to calculate the reverberation times. The software employs pseudo random noise (MLS) as a measurement signal. The measurement setup diagram is shown in Fig. 2.

During the measurements, impulse responses were recorded for the stationary turntable with the base plate

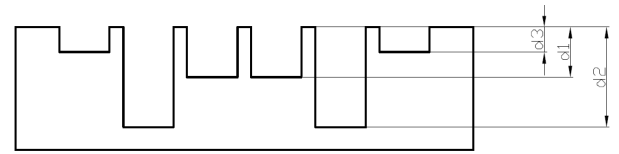

Fig. 5. Cross section of a sample based on a quadratic residue sequence. alone and with the sample (Fig. 6). The measurements were used to calculate the reverberation times $T_{1}$ and $T_{2}$. Then the impulse responses were determined for the rotating turntable, which allowed the calculation of the $T_{3}$ times - for the rotating base plate, and the $T_{4}$ times for the rotating sample. In order to obtain an impulse response for exactly one complete rotation of the system studied, it was necessary to synchronise the angle of the turntable rotation with the measurement system.

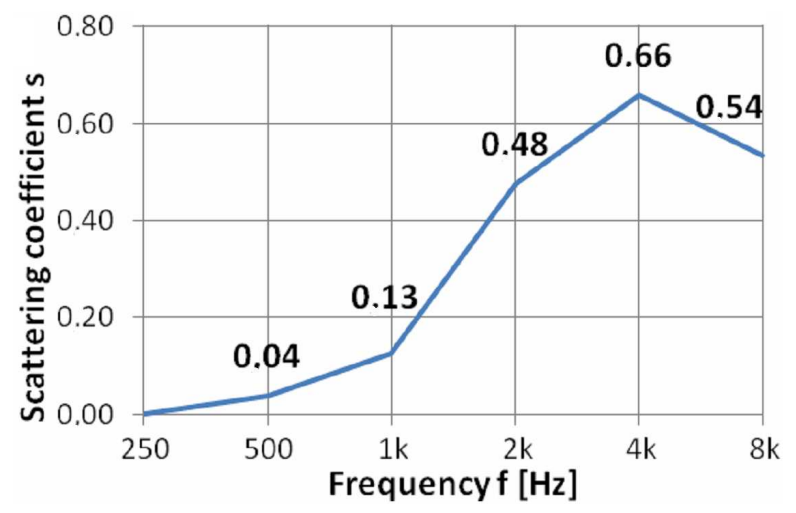

Fig. 6. Sound scattering coefficient as a function of frequency of the system studied.

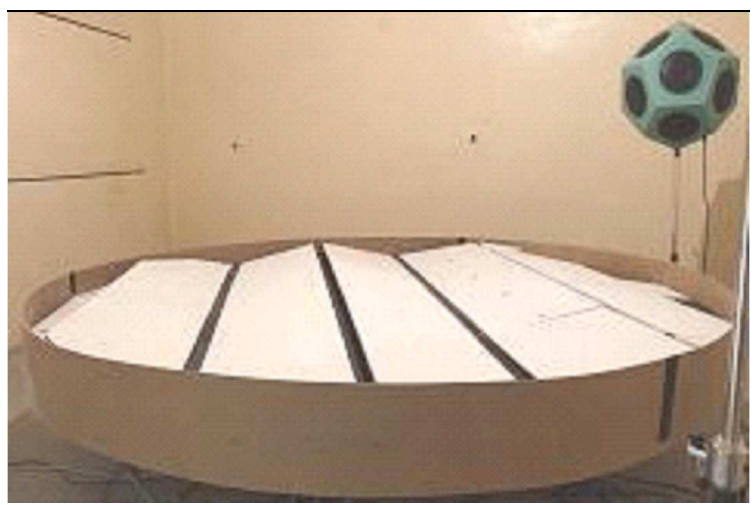

Fig. 7. A sample in the measurement system in a reverberation chamber.

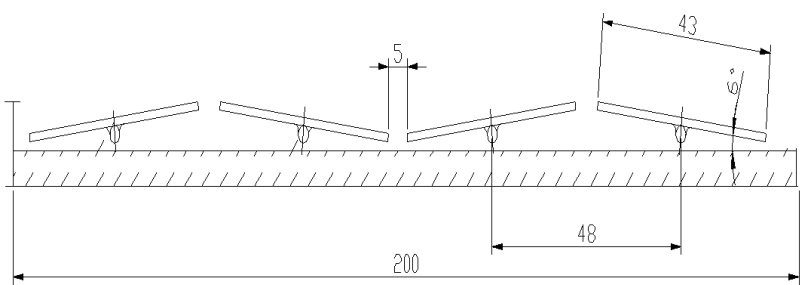

Fig. 8. Cross section of the diffuser used in the experimental rehearsal room.

The impulse responses were determined for two positions of the sound source and for three positions of the 


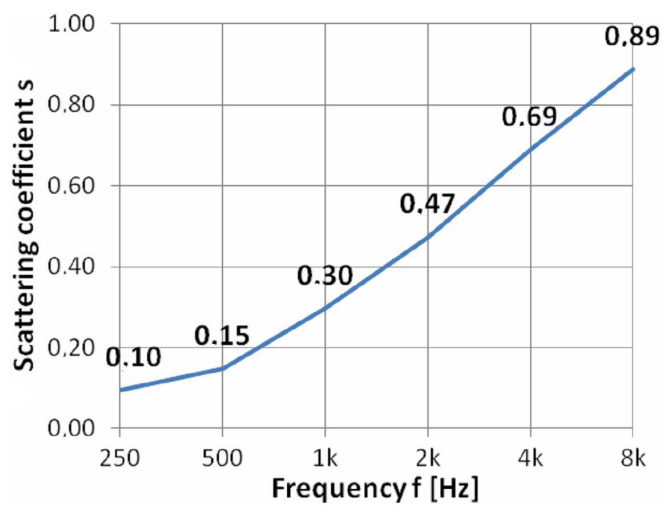

Fig. 9. Sound scattering coefficient of the ceiling diffuser as a function of frequency.

microphone. Figure 6 shows the values of the sound scattering coefficient $s$ as a function of frequency, which were obtained from reverberation times determined from the impulse responses recorded.

The acoustic system described above was proposed as an element of the rear wall equipment of the Lviv Opera hall to improve acoustic performance of the under-balcony space [3].

A similar technique was employed to study the scattering system (diffuser) designed for an experimental rehearsal room for actors. The geometry of the diffuser installed under the ceiling was adapted to the ceiling structure and the room's function. Figures 7 and 8 show the view and a cross section of the diffuser, whereas Fig. 9 shows the sound scattering coefficient found in laboratory studies.

Table shows the results of the study to determine the absorption and the scattering coefficients for the ceiling diffuser. Note that the scattering coefficient has high values for frequencies above $2 \mathrm{kHz}$, whereas the absorption coefficient prevails for medium and low frequencies. This type of diffuser, when installed on large areas of the ceiling, ensured a balanced reverberation time in the room and a uniform distribution of the acoustic field for any interior design.

TABLE

The absorption [5] and the scattering coefficients [1] of the ceiling-mounted diffuser.

\begin{tabular}{c|c|c|c|c|c|c}
\hline \hline$f[\mathrm{~Hz}]$ & 250 & 500 & 1000 & 2000 & 4000 & 8000 \\
\hline$\alpha$ & 0.59 & 0.49 & 0.35 & 0.30 & 0.28 & 0.20 \\
$s[-]$ & 0.10 & 0.15 & 0.30 & 0.47 & 0.69 & 0.89
\end{tabular}

\section{Summary}

The sound scattering coefficient $s$ makes it possible to quantitatively characterize the scattering properties of a surface. Due to its simple physical interpretation, the scattering coefficient $\mathrm{s}$ is used, in addition to the sound absorption coefficient, in the software designed to predict the acoustic properties of interiors. It ensures better correlation between simulation results and the parameters measured in real rooms. But in spite of the simple interpretation, the determination of the scattering coefficient $s$ is time-consuming and requires a properly equipped measurement laboratory. In order to obtain reproducible measurements, the microphones and the sound source must be positioned with high accuracy. It is also necessary to use a turntable that does not generate acoustic disturbances during measurement, and one with an adequately high torque ensuring uniform rotation speed of the sample. Significant problems are also caused by the need to maintain a constant temperature, humidity, and to reduce air movement during measurement. This reduces the measurement time and any operations involving the presence of people in the chamber. One limitation of the method is that it can only perform measurements on structures with a low sound absorption coefficient, i.e., one below 0.5 .

\section{References}

[1] ISO 17497-1:2004. Acoustics - Sound-scattering properties of surfaces - Part 1: Measurement of the random-incidence scattering coefficient in a reverberation room.

[2] J. Cox, P. D‘Antonio, Acoustic Absorbers and Diffusers. Spon Press, London and New York 2004.

[3] T. Kamisiński, R. Kinasz, J. Rubacha, A. Pilch, Czasopismo Techniczne, Architektura Politechniki Krakowskiej, Kraków 2009.

[4] Bruel\&Kjaer DIRAC Room Acoustics Software type 7841, user manual. V. 4.0 (2007).

[5] ISO 354:2003. Acoustics - Measurement of sound absorption in a reverberation room. 